\title{
The MITC9 shell element in plate bending: mathematical analysis of a simplified case
}

\author{
Klaus-Jürgen Bathe · Franco Brezzi • \\ L. Donatella Marini
}

Received: 13 October 2010 / Accepted: 8 December 2010 / Published online: 4 January 2011

(C) Springer-Verlag 2010

\begin{abstract}
We consider the 9-node shell element referred to as the MITC9 shell element in plate bending solutions and present a simplified mathematical analysis. The element uses bi-quadratic interpolations of the rotations and transverse displacement, and the "rotated Raviart-Thomas" interpolations for the transverse shear stresses. A rigorous mathematical analysis of the element is still lacking, even for the simplified case of plate solutions (that is, flat shells), although the numerical evidence suggests a good and reliable behavior. Here we start such an analysis by considering a very simple particular case; namely, a rectangular plate, clamped all around the boundary, and solved with a uniform decomposition. Moreover, we consider only the so-called limit case, corresponding to the limit equations that are obtained for the thickness $t$ going to zero. While the mathematical analysis of the limit case is simpler, such analysis, in general, gives an excellent indication of whether shear locking is present in
\end{abstract}

\footnotetext{
K. J. Bathe ( $\square)$

MIT, Department of Mechanical Engineering,

Massachusetts Institute of Technology, Cambridge,

MA 02139, USA

e-mail: kjb@mit.edu

F. Brezzi · L. D. Marini

Istituto di Matematica Applicata e Tecnologie Informatiche

(IMATI), Consiglio nazionale delle Ricerche (CNR),

Via Ferrata 5/A, 27100 Pavia, Italy

F. Brezzi

Centro di Simulazione Numerica Avanzata (CeSNA), Instituto Universitario di Studi Superiori (IUSS),

Lungoticino Sforza 56, 27100 Pavia, Italy

L. D. Marini

Dipartimento di Matematica, Università di Pavia,

Via Ferrata 5/A, 27100 Pavia, Italy
}

the real case $t>0$. We detail that the element in the setting considered shows indeed optimal behavior.

Keywords Plate $\cdot$ Shell $\cdot$ Shear locking $\cdot$ MITC9 shell element $\cdot$ Convergence analysis

\section{Introduction}

Numerous finite elements based on Kirchhoff plate theory and Reissner-Mindlin plate theory have been proposed for linear plate analyses, see for example $[1,2]$. Today, plate bending elements are available that have been proven to be optimal by mathematical analysis and have revealed that optimal behavior also in numerical solutions, see e.g. [3-6]. Although these elements are available, of course, there is still important research in the analysis of plates to establish computationally more cost-effective schemes in general, and in particular for composite plate analyses where threedimensional effects can be important.

The situation is quite different in the analysis of shells $[7,8]$. Such structures are much more difficult to analyze and efficient, reliable, and generally applicable shell elements are much more difficult to develop. Indeed, while many shell elements have been proposed, there is still no element available that has been proven mathematically to always perform optimally in all shell analyses and in any mesh used. The reason is that the behavior of shell structures is greatly affected by the curvature of the shell, the boundary conditions, the thickness, and the loading applied [7-10]. Moreover, the limit behaviour of a shell for the thickness going to zero can show, even in quite realistic cases, a discouraging variety of patterns that make a unified analysis extremely difficult, see e.g. [8-15]. While complete convergence proofs are very difficult to achieve, and indeed still out of reach, it is important to recognize that mathematical analysis has been extremely valuable 
Fig. 1 Clamped plate considered and finite element results obtained using the MITC9 shell element. a Square plate problem solved, uniform pressure is applied,

$L_{1}=L_{2}=L=1.0$, Young's modulus $=1.7472 \times 10^{7}$, Poisson's ratio $=0.30$.

b Typical mesh used for region A-B-C-D, $N=4$
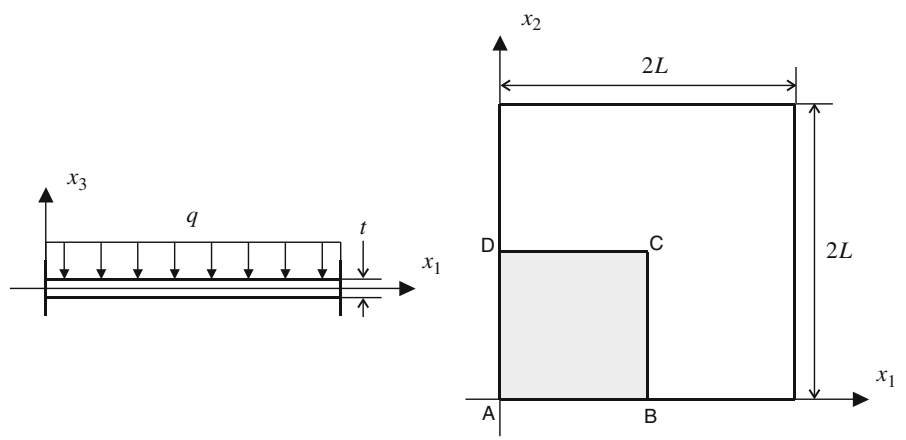

(a)

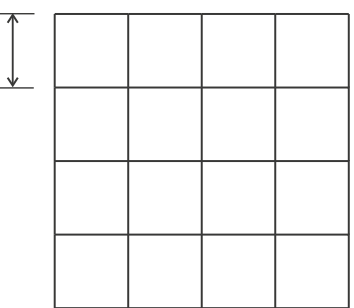

(b)
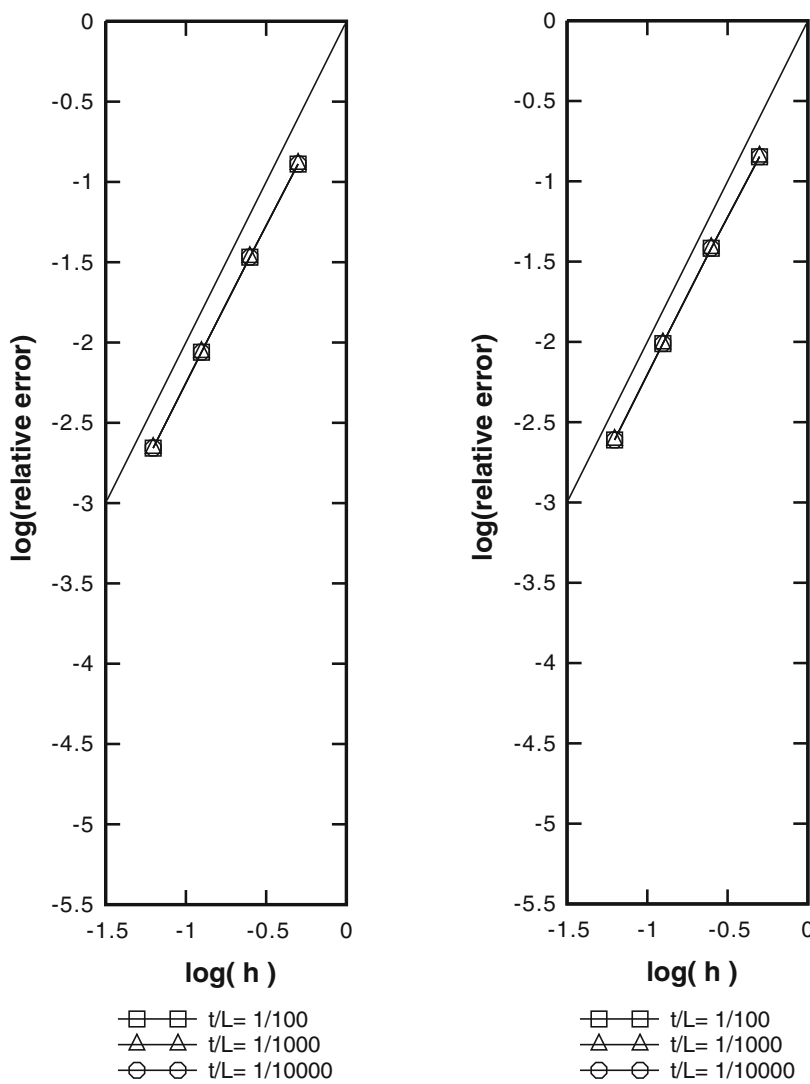

Fig. 2 Results obtained for the clamped plate using the MITC9 shell element. Left relative error for rotations in the $H^{1}$ norm. Right relative error for transverse displacement in the $H^{1}$ norm. In both cases the slope is 2 , as expected

mathematical analysis shows that the convergence of the element is indeed optimal-hence, that the numerical results in Ref. [21] should be expected.

The given analysis and results are valuable because the mathematical proofs give strength to the numerical findings, and the analysis yields insight that may provide a basis for more comprehensive mathematical studies of the element, also directed to the solution of complex shell structures.

In the paper, we will use the notation $A \lesssim B$ whenever there exists a constant $C$, depending only on the ratio of the 
two edges of the rectangular domain, such that $A \leq C B$. Clearly $A \gtrsim B$ will be used whenever $B \lesssim A$.

Throughout the paper, for $s$ an integer we will denote by $H^{s}(\Omega)$ the usual Sobolev space of functions that are squareintegrable in $\Omega$ together with their (weak) derivatives up to the order $s$. We denote by $H_{0}^{s}(\Omega)$ the subspace of $H^{s}(\Omega)$ made of functions that vanish at the boundary $\partial \Omega$ of $\Omega$ together with all their derivatives up to the order $s-1$. Moreover $\|\cdot\|_{s, \Omega}$ or simply $\|\cdot\|_{s}$ will be used to indicate the usual Sobolev norm in $H^{s}(\Omega)$ (or copies of it). In particular we shall use the notation $\|\cdot\|_{0}$ and $(\cdot, \cdot)_{0}$ for the norm and the scalar product (respectively) in $L^{2}(\Omega)$ or copies of it.

\section{Formulation of the problem and basic notation}

In this section we formulate the continuous problem and introduce some required notation for our analysis.

\subsection{The continuous problem}

Let $\Omega$ be the rectangle $] 0, L_{1}[\times] 0, L_{2}[$. Without loss of generality we can assume that $L_{1} \leq L_{2}$. Introducing the spaces

$\Theta:=\left(H_{0}^{1}(\Omega)\right)^{2}, \quad W:=H_{0}^{1}(\Omega)$,

corresponding to hard clamped boundary conditions (see, e.g., [1]), we set

$\mathcal{U}:=\boldsymbol{\Theta} \times W$

with the norm

$\|V\|_{\mathcal{U}}^{2}:=\|\boldsymbol{\eta}\|_{1, \Omega}^{2}+\|v\|_{1, \Omega}^{2} \quad$ for $V=(\boldsymbol{\eta}, v) \in \mathcal{U}$.

We also define, for $\boldsymbol{\eta} \in \boldsymbol{\Theta}$, the symmetric gradient $\boldsymbol{\varepsilon}(\boldsymbol{\eta})$

$(\varepsilon(\boldsymbol{\eta}))_{i, j}:=\frac{1}{2}\left(\frac{\partial \boldsymbol{\eta}_{i}}{\partial x_{j}}+\frac{\partial \boldsymbol{\eta}_{j}}{\partial x_{i}}\right)$,

and for every $t>0$ we define the bilinear form $\mathcal{A}_{t}$ on $\mathcal{U} \times \mathcal{U}$, with Young's modulus equal to 12 and Poisson's ratio equal to 0 , as [24]

$\mathcal{A}_{t}(U, V):=(\boldsymbol{\varepsilon}(\boldsymbol{\theta}), \boldsymbol{\varepsilon}(\boldsymbol{\eta}))_{0}+6 t^{-2}(\nabla w-\boldsymbol{\theta}, \nabla v-\boldsymbol{\eta})_{0}$

for $U=(\boldsymbol{\theta}, w)$ and $V=(\eta, v)$. We are interested in the case " $t$ small". Hence, even when it is not explicitly specified, we will always consider the case of $t$ being small, certainly $t<L_{1}$

The bending part of the bilinear form $\mathcal{A}_{t}$ will be usually denoted by $\mathcal{A}_{b}$, that is

$\mathcal{A}_{b}(U, V):=(\boldsymbol{\varepsilon}(\boldsymbol{\theta}), \boldsymbol{\varepsilon}(\boldsymbol{\eta}))_{0}$.

We recall the following well known result (see, e.g., $[4,8]$ ).
Proposition 2.1 The bilinear form $\mathcal{A}_{t}$ is continuous and elliptic on $\mathcal{U} \times \mathcal{U}$ for every $t>0$, in the sense that: for every $t$ with $0<t<L_{1}$ there exist three positive constants $C_{t}, C_{b}$ and $C_{s}$ such that

$\mathcal{A}_{t}(U, V) \leq C_{t}\|U\|_{\mathcal{U}}\|V\|_{\mathcal{U}} \quad \forall U, V \in \mathcal{U}$

$\mathcal{A}_{b}(U, V) \leq C_{b}\|U\|_{\mathcal{U}}\|V\|_{\mathcal{U}} \quad \forall U, V \in \mathcal{U}$

$\mathcal{A}_{t}(V, V) \geq C_{s}\|V\|_{\mathcal{U}}^{2} \quad \forall V \in \mathcal{U}$.

Moreover $C_{b}$ and $C_{s}$ are independent of $t$.

Proof The continuity properties (2.7) and (2.8) are obvious. Next, we recall the Korn inequality: for $V=(\eta, v) \in \mathcal{U}$

$\mathcal{A}_{b}(V, V) \equiv(\boldsymbol{\varepsilon}(\boldsymbol{\eta}), \boldsymbol{\varepsilon}(\boldsymbol{\eta}))_{0} \gtrsim\|\boldsymbol{\eta}\|_{1, \Omega}^{2}$,

which implies

$\mathcal{A}_{t}(V, V) \geq \mathcal{A}_{b}(V, V) \gtrsim\|\boldsymbol{\eta}\|_{1, \Omega}^{2}$,

and since $L_{1}>t>0$ we obviously have

$\|\nabla v\|_{0, \Omega}^{2} \leq 2\|\nabla v-\eta\|_{0, \Omega}^{2}+2\|\eta\|_{0, \Omega}^{2} \lesssim \mathcal{A}_{t}(V, V)$,

and the property in (2.9) follows from (2.11) and (2.12).

Remark 2.2 For simplicity we assumed in (2.5) Poisson's ratio equal to 0 . However, it is clear that the properties of $\mathcal{A}_{t}$ and $\mathcal{A}_{b}$ in the above proposition (which we use for all our estimates) also hold if the bilinear forms include the effects of nonzero Poisson's ratio.

We now fix a function (load) $g \in L^{2}(\Omega)$ and define

$(G, V)_{0}:=(g, v)_{0} \quad$ for $V=(\boldsymbol{\eta}, v)$,

and we note that, obviously,

$\left|(G, V)_{0}\right| \leq\|g\|_{0}\|V\|_{\mathcal{U}}$.

We then consider the problem:

$\left\{\begin{array}{l}\text { Find } U \in \mathcal{U} \text { such that } \\ \mathcal{A}_{t}(U, V)=(G, V)_{0} \quad \forall V \in \mathcal{U} .\end{array}\right.$

From Proposition 2.1 we have immediately existence and uniqueness of the solution of (2.15).

Proposition 2.3 For every $t>0$ and for every $g \in L^{2}(\Omega)$ problem (2.15) has a unique solution $U$ that satisfies

$\|U\|_{\mathcal{U}} \lesssim\|g\|_{0}$.

Moreover, $U$ coincides with the unique minimizer on $\mathcal{U}$ of the functional

$J_{t}^{R M}(V):=\frac{1}{2} \mathcal{A}_{t}(V, V)-(G, V)_{0}$. 
2.2 The Lagrange multipliers formulation

We introduce now the formulation with multipliers. For this we need to define the spaces

$H(\operatorname{rot} ; \Omega):=\left\{\delta \in\left(L^{2}(\Omega)\right)^{2}\right.$, such that $\left.\operatorname{rot} \delta \in L^{2}(\Omega)\right\}$,

where $\operatorname{rot} \delta$, as usual in two-dimensional settings, is defined as $\operatorname{rot} \delta:=\frac{\partial \delta_{2}}{\partial x_{1}}-\frac{\partial \delta_{1}}{\partial x_{2}}$, and

$Q:=H_{0}(\operatorname{rot} ; \Omega) \equiv\{\boldsymbol{\delta} \in H(\operatorname{rot} ; \Omega)$ such that,

$$
\boldsymbol{\delta} \cdot \mathbf{t}=0 \text { on } \partial \Omega\}
$$

where $\mathbf{t}$ is the unit counterclockwise tangent vector to $\partial \Omega$. We also define the norm

$\|\boldsymbol{\delta}\|_{Q}^{2}:=\|\boldsymbol{\delta}\|_{0, \Omega}^{2}+\|\operatorname{rot} \boldsymbol{\delta}\|_{0, \Omega}^{2}$.

We can also define the space of multipliers $\mathcal{M}$ as

$\mathcal{M}:=Q^{\prime}$

(that is, the dual space of $Q$ ). We shall often use the following notation

$\mathcal{L}:=\left(L^{2}(\Omega)\right)^{2}$

It is evident that $Q \subseteq \mathcal{L}$ with continuous dense embedding so that $\mathcal{L}$ (that we identify as usual with its own dual space) can be identified with a dense subspace of $\mathcal{M}=Q^{\prime}$.

Remark 2.4 It can be proved (see, e.g., [4]) that

$\mathcal{M} \equiv\left\{\boldsymbol{\mu} \in\left(H^{-1}(\Omega)\right)^{2}\right.$ such that $\left.\operatorname{div} \boldsymbol{\mu} \in H^{-1}(\Omega)\right\}$.

It will also be convenient to introduce the operator $B$ : $\mathcal{U} \rightarrow Q$ defined as

$B(V)=\nabla v-\eta \quad$ for $V=(\eta, v)$.

The following result is well known (see e.g. [4]).

Proposition 2.5 The space $Q$ coincides with the image of $\mathcal{U}$ through the operator B. Moreover, for every $\delta \in Q$ there exists a $V \in \mathcal{U}$ such that $B(V)=\delta$ and

$\|V\|_{\mathcal{U}} \lesssim\|\delta\|_{Q}$

We consider now the saddle-point problem:

$\left\{\begin{array}{l}\text { Find } U \equiv(\boldsymbol{\theta}, w) \in \mathcal{U} \text { and } \lambda \in \mathcal{L} \text { such that } \\ \mathcal{A}_{b}(U, V)+\mathcal{M}\langle\lambda, B(V)\rangle_{Q}=(G, V)_{0} \quad \forall V \in \mathcal{U} \\ \mathcal{M}\langle\boldsymbol{\mu}, B(U)\rangle_{Q}-\frac{t^{2}}{6}(\boldsymbol{\lambda}, \boldsymbol{\mu})_{0}=0 \quad \forall \boldsymbol{\mu} \in \mathcal{L} .\end{array}\right.$
As Proposition 2.5 implies the inf-sup condition

$\exists \beta>0$ such that $\inf _{\boldsymbol{\mu} \in \mathcal{M}} \sup _{V \in \mathcal{U}} \frac{Q\langle B(V), \boldsymbol{\mu}\rangle_{\mathcal{M}}}{\|V\|_{\mathcal{U}}\|\boldsymbol{\mu}\|_{\mathcal{M}}} \geq \beta$

we can easily have the following result $[4,25]$.

Proposition 2.6 For every $t>0$ and for every $g \in L^{2}(\Omega)$, problem (2.26) has a unique solution $(U, \lambda)$ that satisfies

$\|U\|_{\mathcal{U}}+\|\lambda\|_{\mathcal{M}} \lesssim\|g\|_{0}$.

Moreover, $U$ coincides with the solution of (2.15).

Proof The result is rather classical, but we summarize the proof for the convenience of the reader. Let us first consider, for every $t>0$, the solution $U$ of (2.15), and set $\lambda:=6 t^{-2} B(U)$ that belongs to $Q \subset \mathcal{L}$. It is easy to see that the pair $(U, \lambda)$ is a solution of (2.26). Moreover the first part of (2.28), that is

$\|U\|_{\mathcal{U}} \lesssim\|g\|_{0}$

holds true due to (2.16). Finally, from (2.27) we immediately have that

$\exists V^{*} \in \mathcal{U}$ such that $\|\lambda\|_{\mathcal{M}} \leq \frac{1}{\beta} \frac{Q\left\langle B\left(V^{*}\right), \lambda\right\rangle_{\mathcal{M}}}{\left\|V^{*}\right\|_{\mathcal{U}}}$,

and using the first equation of (2.26) with $V=V^{*}$, and then (2.14) and (2.8), we have

$\|\lambda\|_{\mathcal{M}} \leq \frac{1}{\beta} \frac{\left(G, V^{*}\right)_{0}-\mathcal{A}_{b}\left(U, V^{*}\right)}{\left\|V^{*}\right\|_{\mathcal{U}}} \lesssim\left(\|g\|_{0}+\|U\|_{\mathcal{U}}\right)$,

and the result follows from (2.29) and (2.31).

Remark 2.7 In mathematical terms, problem (2.26) is singularly perturbed, since, for a general $G \in \mathcal{U}^{\prime}$ and a general Lipschitz domain, its solution $(U(t), \lambda(t))$ exists in $\mathcal{U} \times \mathcal{L}$ for every $t>0$, but is not uniformly bounded in $\mathcal{U} \times \mathcal{L}$. However, $U(t)$ is uniformly bounded in $\mathcal{U}$, and the inf-sup condition (2.27) provides a uniform bound for $\lambda(t)$ in $\mathcal{M}$ (although not in $\mathcal{L}$ ).

\subsection{The limit problem}

As the bound in (2.28) does not depend on $t$ (and $\mathcal{U}$ and $\mathcal{M}$ are Hilbert spaces), it is easy to see and well known (see e.g. [4], Theorem VII.3.1) that $U=U(t)$ and $\lambda=\lambda(t)$ have a limit for $t \rightarrow 0$.

Proposition 2.8 For every $g \in L^{2}(\Omega)$ we have

$\lim _{t \rightarrow 0}(U(t), \lambda(t))=\left(U_{0}, \lambda_{0}\right)$

where $\left(U_{0}, \lambda_{0}\right)$ is the unique solution of the limit problem

$\left\{\begin{array}{l}\text { Find } U_{0} \equiv\left(\boldsymbol{\theta}_{0}, w_{0}\right) \in \mathcal{U} \text { and } \lambda_{0} \in \mathcal{M} \text { such that } \\ \mathcal{A}_{b}\left(U_{0}, V\right)+\mathcal{M}\left\langle\lambda_{0}, B(V)\right\rangle_{Q}=(G, V)_{0} \quad \forall V \in \mathcal{U} \\ \mathcal{M}\left\langle\boldsymbol{\mu}, B\left(U_{0}\right)\right\rangle_{Q}=0 \quad \forall \boldsymbol{\mu} \in \mathcal{M} .\end{array}\right.$ 
Fig. 3 The displacement along the mid-line of the plate of

Fig. 1 is shown for $N=4,16$, 32 and different values of plate thickness for the MITC4 element; the normalizing factor is $q /\left(10^{8} * t^{3}\right)$. For $t / L$ smaller than $1 / 100$ the displacements are practically equal to those of the case $t / L=1 / 100$

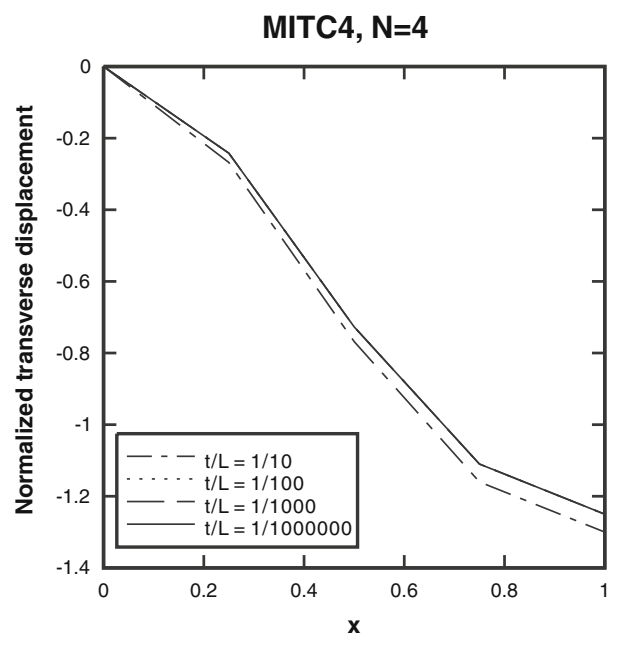

MITC4, $\mathrm{N}=16$

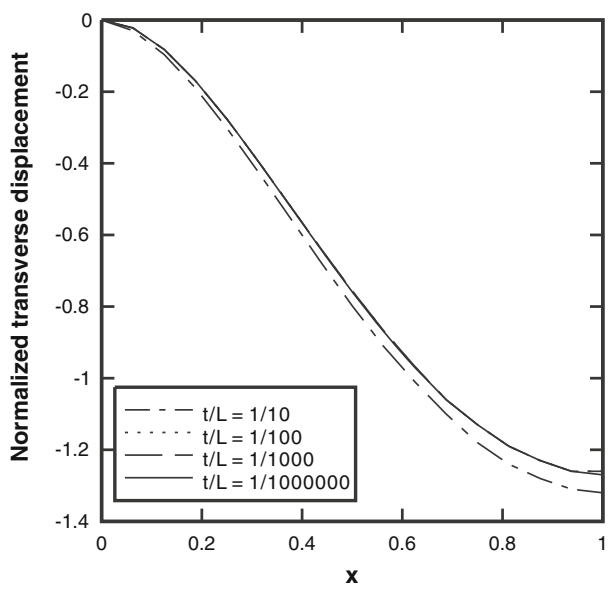

MITC4, $\mathrm{N}=32$

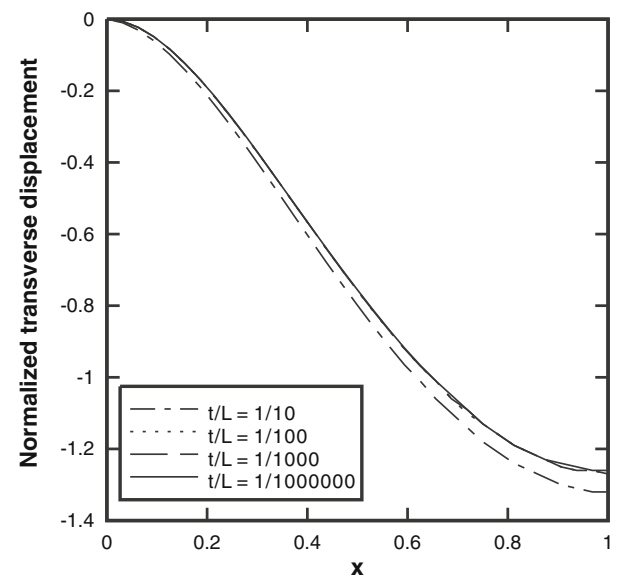

Moreover, $\boldsymbol{\theta}_{0}=\nabla w_{0}$ and $w_{0}$ is the solution of the (Kirchhoff-like) problem

$\Delta^{2} w_{0}=g$ in $\Omega, \quad w_{0}=0$ and $\frac{\partial w_{0}}{\partial n}=0$ on $\partial \Omega$.

It is immediate to see that, introducing the linear space

$\mathcal{Z}=\{V=(v, \eta) \in \mathcal{U}$ such that $\boldsymbol{\eta}=\nabla v\}$,

problem (2.33) is equivalent to

$$
\left\{\begin{array}{l}
\text { Find } U_{0} \in \mathcal{Z} \text { such that } \\
\mathcal{A}_{b}\left(U_{0}, V\right)=(G, V)_{0} \quad \forall V \in \mathcal{Z}
\end{array}\right.
$$

in the sense that: if $\left(U_{0}, \lambda_{0}\right)$ solves $(2.33)$ then $U_{0}$ solves (2.36).

We shall focus on this limit problem in our convergence analysis of the MITC9 shell element. This analysis is tractable and will give a strong indication on whether the element is shear-locking or not when solving this problem using the specified uniform decompositions.

Figures 3 and 4 give the profiles of the transverse displacement of the plate along its midline, as calculated using the MITC4 shell element-which is in this solution identical to the MITC4 plate element and known to be lockingfree [24] - and the 4-node displacement-based elementwhich is known to severely lock. We see that for the MITC4 shell element, the displacements are indistinguishable for the coarse and fine meshes used when $t / L=1 / 100$ and smaller. Shear deformations contribute to the response when the plate is thick. On the other hand, for the displacementbased element, the displacements become rapidly small when the thickness of the plate decreases, that is, the element locks. These numerical results, together with those of Fig. 2, illustrate that - as expected - in a mathematical analysis it is reasonable to consider the limit problem as a valuable indicator of the performances of an element for thin and moderately thick plates.

\section{The discretized problem}

We consider now, for simplicity, a sequence of decompositions $\mathcal{T}_{h}$ of our domain $\Omega$ into rectangles $K$ by means of the points 
Fig. 4 The displacement along the mid-line of the plate of

Fig. 1 is shown for $N=4,16$, 32 and different values of plate thickness for the 4-node displacement-based element, here referred to as DISP4; the normalizing factor is $q /\left(10^{8} * t^{3}\right)$. For $t / L$ smaller than $1 / 100$ the displacements are extremely small
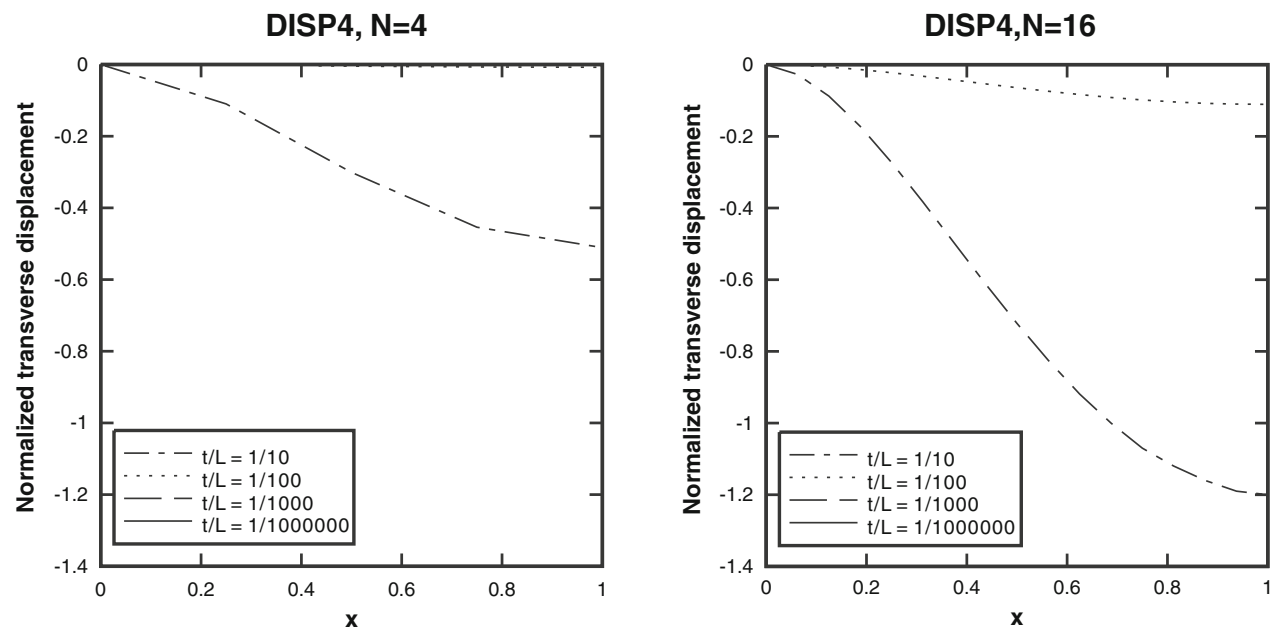

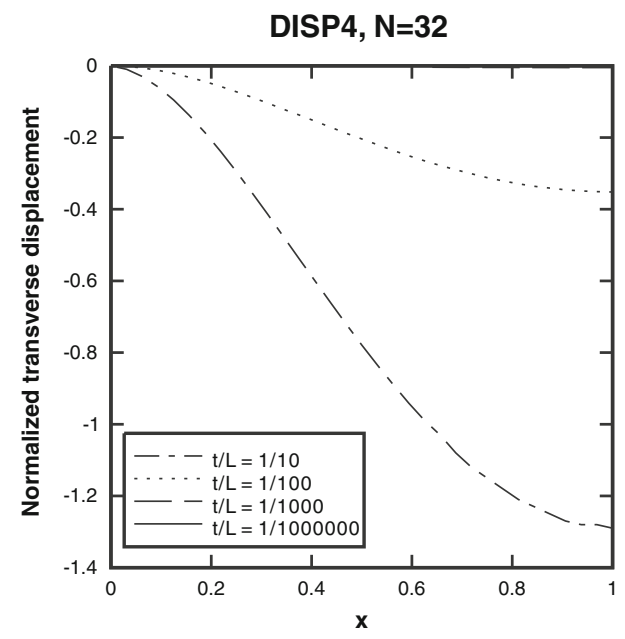

$0 \equiv x_{0}<x_{1}<\cdots<x_{I} \equiv L_{1} \quad 0 \equiv y_{0}<y_{1}<\cdots<y_{J} \equiv L_{2}$

and we set as usual

$h_{x}:=\max _{0 \leq i \leq I-1}\left(x_{i+1}-x_{i}\right) \quad h_{y}:=\max _{0 \leq j \leq J-1}\left(y_{j+1}-y_{j}\right)$

$h:=\max \left\{h_{x}, h_{y}\right\}$.

For $r$ and $s$ integers $\geq 0$ we define the space $\mathbb{Q}_{r, s}$ as the space of polynomials of degree $\leq r$ in $x_{1}$ and of degree $\leq s$ in $x_{2}$. When $r=s$ we will just, as usual, write $\mathbb{Q}_{r}$ instead of $\mathbb{Q}_{r, r}$. Then we consider the finite element spaces

$\boldsymbol{\Theta}^{h}:=\left\{\boldsymbol{\theta} \in \boldsymbol{\Theta}\right.$ such that $\left.\forall K \in \mathcal{T}_{h}, \quad \boldsymbol{\theta} \in \mathbb{Q}_{2}(K)\right\}$,

$W^{h}:=\left\{v \in W\right.$ such that $\left.\forall K \in \mathcal{T}_{h}, \quad v \in \mathbb{Q}_{2}(K)\right\}$,

and

$\mathcal{M}^{h}:=\left\{\boldsymbol{\mu}\right.$ such that $\left.\boldsymbol{\mu}_{\mid K} \in \mathbb{Q}_{1,2} \times \mathbb{Q}_{2,1} \forall K \in \mathcal{T}_{h}\right\}$.

Finally, we define the reduction operator $\Pi$ from $\left(C^{0}(\bar{\Omega})\right)^{2}$ to $\mathcal{M}^{h}$. For this, on every interval $\left(x_{i}, x_{i+1}\right)$ we define the midpoint $x_{i}^{m}$ and the two zeroes of the second degree
Legendre polynomial $x_{i}^{\ell 1}$ and $x_{i}^{\ell 2}$; similarly, for every interval $\left(y_{j}, y_{j+1}\right)$ we define the midpoint $y_{j}^{m}$ and the two zeroes of the second degree Legendre polynomial $y_{j}^{\ell 1}$ and $y_{j}^{\ell 2}$ (see Fig. 5). Then, for every $\chi \in\left(C^{0}(\bar{\Omega})\right)^{2}$ we define $\Pi \chi$ as the unique $\boldsymbol{\mu} \in \mathcal{M}^{h}$ such that (see Fig. 6)

$\mu_{1}\left(x_{i}^{\ell 1}, y_{j}\right)=\chi_{1}\left(x_{i}^{\ell 1}, y_{j}\right) \quad i=0, \ldots, I-1, \quad j=0, \ldots, J$

$\mu_{1}\left(x_{i}^{\ell 2}, y_{j}\right)=\chi_{1}\left(x_{i}^{\ell 2}, y_{j}\right) \quad i=0, \ldots, I-1, \quad j=0, \ldots, J$

$\mu_{1}\left(x_{i}^{\ell 1}, y_{j}^{m}\right)=\chi_{1}\left(x_{i}^{\ell 1}, y_{j}^{m}\right) \quad i=0, \ldots, I-1, \quad j=0, \ldots, J-1$

$\mu_{1}\left(x_{i}^{\ell 2}, y_{j}^{m}\right)=\chi_{1}\left(x_{i}^{\ell 2}, y_{j}^{m}\right) \quad i=0, \ldots, I-1, \quad j=0, \ldots, J-1$

and

$$
\begin{array}{ll}
\mu_{2}\left(x_{i}, y_{j}^{\ell 1}\right)=\chi_{2}\left(x_{i}, y_{j}^{\ell 1}\right) & i=0, \ldots, I, \quad j=0, \ldots J-1 \\
\mu_{2}\left(x_{i}, y_{j}^{\ell 2}\right)=\chi_{2}\left(x_{i}, y_{j}^{\ell 2}\right) & i=0, \ldots, I, \quad j=0, \ldots J-1 \\
\mu_{2}\left(x_{i}^{m}, y_{j}^{\ell 1}\right)=\chi_{2}\left(x_{i}^{m}, y_{j}^{\ell 1}\right) & i=0, \ldots, I-1, \quad j=0, \ldots J-1 \\
\mu_{2}\left(x_{i}^{m}, y_{j}^{\ell 2}\right)=\chi_{2}\left(x_{i}^{m}, y_{j}^{\ell 2}\right) & i=0, \ldots, I-1, \quad j=0, \ldots J-1
\end{array}
$$




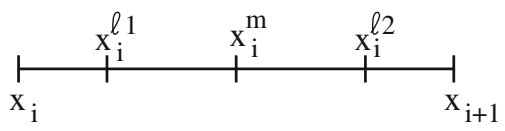

Fig. 5 Midpoint and two Gauss points for 2-point integration on an interval
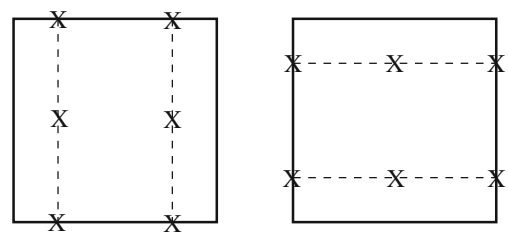

Fig. 6 D.o.f. for $\mu_{1}$ (left) and $\mu_{2}$ (right)

It is important to note that for every $v \in W^{h}$ we have $\nabla v \in$ $\mathcal{M}^{h}$, so that

$\Pi(\nabla v)=\nabla v \quad \forall v \in W^{h}$.

Moreover, owing to the (obvious) continuity of $\Pi$ in finite dimensional spaces made of piecewise smooth functions we easily have

$\|\Pi \boldsymbol{\eta}\|_{0} \lesssim\|\boldsymbol{\eta}\|_{0} \quad \forall \boldsymbol{\eta} \in \mathbf{\Theta}^{h}$

We can now set $\mathcal{U}^{h}:=\boldsymbol{\Theta}^{h} \times W^{h}$ and consider the discrete problem:

$\left\{\begin{array}{l}\text { Find } U^{h} \equiv\left(\boldsymbol{\theta}^{h}, w^{h}\right) \in \mathcal{U}^{h}, \text { and } \lambda^{h} \in \mathcal{M}^{h} \text { such that } \\ \mathcal{A}_{b}\left(U^{h}, V\right)+\left(\lambda^{h}, \Pi(B(V))\right)_{0}=(G, V)_{0} \quad \forall V \in \mathcal{U}^{h} \\ \left(\boldsymbol{\mu}, \Pi\left(B\left(U^{h}\right)\right)\right)_{0}-\frac{t^{2}}{6}\left(\lambda^{h}, \boldsymbol{\mu}\right)_{0}=0 \quad \forall \boldsymbol{\mu} \in \mathcal{M}^{h} .\end{array}\right.$

Existence and uniqueness of the solution of the discrete problem for $t>0$ follow exactly as for the continuous problem.

Remark 3.1 The corresponding MITC9 plate element uses the same space $\boldsymbol{\Theta}^{h}$ as in (3.3), while $W_{h}$ consists of local 8 -node serendipity functions. The space $\mathcal{M}$ is more sophisticated: the first component is made of local $\mathbb{P}_{2}$ polynomials without the monomial $x^{2}$, while the second component is made of local $\mathbb{P}_{2}$ polynomials without the monomial $y^{2}$ [6]. The analysis (see e.g. [26]) is largely based on the properties of the $\mathbb{Q}_{2}-\mathbb{P}_{1}$ Stokes element. The operator $\Pi$ uses, for each component, the average over the element domain instead of the two internal stations shown in Fig. 6.

Remark 3.2 We should note that the bending part in (3.10) is not reduced, like also for the MITC9 plate element. In the MITC9 shell element the membrane and the bending strains are actually reduced in order to circumvent membrane locking. However, this reduction does not affect possible shear locking and the convergence rate of the element in the plate solutions considered here.
We now consider and analyze the limit problem of (3.10) for $t \rightarrow 0$. Introducing the subspace

$\mathcal{Z}^{h}=\left\{V=(v, \boldsymbol{\eta}) \in \mathcal{U}^{h}\right.$ such that $\left.\Pi(\boldsymbol{\eta})=\nabla v\right\}$,

it is immediate to see that, for $t \rightarrow 0, U^{h}(t)$ converges to the solution $U_{0}^{h}$ of the limit problem

$\left\{\begin{array}{l}\text { Find } U_{0}^{h} \in \mathcal{Z}^{h} \text { such that } \\ \mathcal{A}_{b}\left(U_{0}^{h}, V\right)=(G, V)_{0} \quad \forall V \in \mathcal{Z}^{h},\end{array}\right.$

that can be obviously seen as a discretization of the continuous limit problem (2.36). Existence and uniqueness of the solution of (3.12) follow immediately from (2.10). Here we want to study the error $\left\|U_{0}-U_{0}^{h}\right\|_{\mathcal{U}}$.

We start with the following "abstract" result.

Theorem 3.3 Let $\left(U_{0}, \lambda_{0}\right)$ be the solution of (2.33), and let $U_{0}^{h}$ be the solution of (3.12). Let moreover $U^{I}$ be any element of $\mathcal{Z}^{h}$. Then we have

$$
\begin{aligned}
\left\|U_{0}-U_{0}^{h}\right\| \mathcal{U} \lesssim & \left\|U_{0}-U^{I}\right\|_{\mathcal{U}} \\
& +\sup _{V^{h} \in \mathcal{Z}^{h}} \frac{\left(\lambda_{0}, B\left(V^{h}\right)-\Pi\left(B\left(V^{h}\right)\right)\right)}{\left\|V^{h}\right\|_{\mathcal{U}}} .
\end{aligned}
$$

Proof For all $V=(\eta, v) \in \mathcal{Z}^{h}$ we have first, using (2.10)

$\|\boldsymbol{\eta}\|_{1, \Omega}^{2} \lesssim(\boldsymbol{\varepsilon}(\boldsymbol{\eta}), \boldsymbol{\varepsilon}(\boldsymbol{\eta})) \lesssim \mathcal{A}_{b}(V, V)$,

and using (3.11), (3.9), and (3.14):

$|\nabla v|_{0, \Omega}^{2}=\|\Pi(\boldsymbol{\eta})\|_{0, \Omega}^{2} \lesssim\|\boldsymbol{\eta}\|_{0, \Omega}^{2} \lesssim \mathcal{A}_{b}(V, V)$,

so that

$\|V\|_{\mathcal{U}}^{2} \lesssim \mathcal{A}_{b}(V, V) \quad \forall V \in \mathcal{Z}^{h}$.

Now we apply (3.16) to $V=U_{0}^{h}-U^{I}$, then we add and subtract $U_{0}$, then we use (3.12) and (2.33) for the first term, and the Cauchy-Schwarz inequality for the second:

$$
\begin{aligned}
\left\|U_{0}^{h}-U^{I}\right\|_{\mathcal{U}}^{2} \lesssim & \mathcal{A}_{b}\left(U_{0}^{h}-U^{I}, U_{0}^{h}-U^{I}\right) \\
= & \mathcal{A}_{b}\left(U_{0}^{h}-U_{0}, U_{0}^{h}-U^{I}\right) \\
& +\mathcal{A}_{b}\left(U_{0}-U^{I}, U_{0}^{h}-U^{I}\right) \\
\lesssim & \left(G, U_{0}^{h}-U^{I}\right) \\
& -\left\{\left(G, U_{0}^{h}-U^{I}\right)-\left(\lambda_{0}, B\left(U_{0}^{h}-U^{I}\right)\right)\right\} \\
& +\left\|U_{0}-U^{I}\right\| \mathcal{U}\left\|U_{0}^{h}-U^{I}\right\|_{\mathcal{U}} \\
= & \left(\lambda_{0}, B\left(U_{0}^{h}-U^{I}\right)\right) \\
& +\left\|U_{0}-U^{I}\right\| \mathcal{U}\left\|U_{0}^{h}-U^{I}\right\|_{\mathcal{U} .}
\end{aligned}
$$

To treat the first term in the last line of (3.17) we remember that $\Pi\left(B\left(U_{0}^{h}-U^{I}\right)\right)=0$ so that

$$
\begin{aligned}
& \left|\left(\lambda_{0}, B\left(U_{0}^{h}-U^{I}\right)\right)\right| \\
& =\left|\left(\lambda_{0}, B\left(U_{0}^{h}-U^{I}\right)-\Pi\left(B\left(U_{0}^{h}-U^{I}\right)\right)\right)\right| \\
& \leq\left(\sup _{V^{h} \in \mathcal{Z}^{h}} \frac{\left(\lambda_{0}, B\left(V^{h}\right)-\Pi\left(B\left(V^{h}\right)\right)\right)}{\left\|V^{h}\right\|_{\mathcal{U}}}\right)\left\|U_{0}^{h}-U^{I}\right\|_{\mathcal{U}} .
\end{aligned}
$$


Inserting (3.18) in (3.17) and simplifying by $\left\|U_{0}^{h}-U^{I}\right\|_{\mathcal{U}}$ we get the result.

\section{Error estimates for the limit problem}

Here we want to apply the general result of Theorem 3.3 to our specific discretization. To this purpose we need an estimate for the two pieces appearing on the right-hand side of (3.13). We start with the first one.

Theorem 4.1 Let $\left(U_{0}, \lambda_{0}\right) \equiv\left(\left(\boldsymbol{\theta}_{0}, w_{0}\right), \lambda_{0}\right)$ be the solution of (2.33). Then there exist $\boldsymbol{\theta}^{I} \in \boldsymbol{\Theta}^{h}$ and $w^{I} \in W^{h}$ such that

$\left\|\boldsymbol{\theta}_{0}-\boldsymbol{\theta}^{I}\right\|_{1, \Omega} \leq C h^{2}\left\|w_{0}\right\|_{4, \Omega}$,

$\left\|w_{0}-w^{I}\right\|_{1, \Omega} \leq C h^{2}\left\|w_{0}\right\|_{3, \Omega}$,

$\Pi \boldsymbol{\theta}^{I}=\nabla w^{I}$

Proof We follow techniques similar to those used in [27].

Define first $\widetilde{w}$ as the Bogner-Fox-Schmit approximation of $w_{0}$ defined locally by:

$\widetilde{w} \in C^{1}(\Omega): \quad \widetilde{w}_{\mid K} \in \mathbb{Q}_{3}(K) \quad \forall K$,

$\widetilde{w}=w_{0}, \widetilde{w}_{x}=\left(w_{0}\right)_{x}$,

$\widetilde{w}_{y}=\left(w_{0}\right)_{y}, \widetilde{w}_{x y}=\left(w_{0}\right)_{x y}$ at the vertices of each $K$,

and recall that, on each $K$,

$\left|\widetilde{w}-w_{0}\right|_{s, K} \leq C h^{r-s}\left|w_{0}\right|_{r, K}, \quad 0 \leq s \leq r \leq 4$.

Next, let $\widetilde{w}^{I} \in W_{h}$ be the classical 9-nodes continuous interpolant of $\widetilde{w}$, defined locally by

$\widetilde{w}^{I} \in \mathbb{Q}_{2}(K) \quad \forall K$,

$\widetilde{w}^{I}=\widetilde{w}$ at the four vertices of each element $K$,

$\widetilde{w}^{I}=\widetilde{w}$ at the midpoints of each edge $e$ of $K, \quad \forall K$,

$\widetilde{w}^{I}=\widetilde{w}$ at the barycenter of each element $K$,

for which the following estimate holds

$\left|\widetilde{w}-\widetilde{w}^{I}\right|_{s, K} \leq C h^{r-s}|\widetilde{w}|_{r, K} \leq C h^{r-s}\left|w_{0}\right|_{r, K}$,

$$
0 \leq s \leq r \leq 3
$$

for all $K$. Setting $w^{I}=\widetilde{w}^{I}$, from (4.5) and (4.7) we have then in particular

$\left\|w_{0}-w^{I}\right\|_{1, \Omega} \leq\left\|w_{0}-\widetilde{w}\right\|_{1, \Omega}+\left\|\widetilde{w}-w^{I}\right\|_{1, \Omega} \leq C h^{2}\left|w_{0}\right|_{3, \Omega}$.

We notice that an alternative, equivalent definition of (4.6) is:

$\widetilde{w}^{I} \in \mathbb{Q}_{2}(K) \quad \forall K$,

$\widetilde{w}^{I}=\widetilde{w}$ at the four vertices of each element $K$,

$\int_{e}\left(\widetilde{w}-\widetilde{w}^{I}\right) d s=0$ on each edge $e$ of $K, \quad \forall K$,

$\int_{K}\left(\widetilde{w}-\widetilde{w}^{I}\right) d x d y=0, \quad \forall K$.
Indeed, by Simpson's integration formula on each edge, exact for polynomials of degree $\leq 3$ we deduce

$\int_{e}\left(\widetilde{w}-\widetilde{w}^{I}\right) d s=0$ on each edge $e$ of $K$.

By applying the tensor-product Simpson rule we also deduce

$\int_{K}\left(\widetilde{w}-\widetilde{w}^{I}\right) d x d y=0 \quad \forall K$.

We define now $\boldsymbol{\theta}^{I} \in \boldsymbol{\Theta}^{h}$ as the continuous interpolant of $\nabla \widetilde{w}$ defined as in (4.6), that is,

$\theta_{1}^{I}=\widetilde{w}_{x}, \quad \theta_{2}^{I}=\widetilde{w}_{y}$ at the 9 nodes.

From (4.5) and (4.7) we deduce

$\left\|\boldsymbol{\theta}_{0}-\boldsymbol{\theta}^{I}\right\|_{1, \Omega}=\left\|\nabla\left(w_{0}-\widetilde{w}\right)+\nabla \widetilde{w}-\boldsymbol{\theta}^{I}\right\|_{1, \Omega} \leq C h^{2}\left|w_{0}\right|_{4, \Omega}$.

In order to prove (4.3), let $K \equiv\left(x_{i}, x_{i+1}\right) \times\left(y_{j}, y_{j+1}\right)$ be an element of $\mathcal{T}_{h}$, let $e\left(x, y_{j}\right):=\widetilde{w}\left(x, y_{j}\right)-\widetilde{w}^{I}\left(x, y_{j}\right)$, and let $p_{1}(x)$ be any polynomial of degree $\leq 1$. Using (4.6) and (4.9) we easily have that $e\left(x, y_{j}\right)$ vanishes at the endpoints and it has zero mean value on $\left(x_{i}, x_{i+1}\right)$. Hence, integrating by parts,

$$
\begin{aligned}
\int_{x_{i}}^{x_{i+1}} e_{x}\left(x, y_{j}\right) p_{1}(x) \mathrm{d} x= & -\int_{x_{i}}^{x_{i+1}} e\left(x, y_{j}\right) p_{1, x}(x) \mathrm{d} x \\
& +\left.e\left(x, y_{j}\right) p_{1}(x)\right|_{x_{i}} ^{x_{i+1}}=0 .
\end{aligned}
$$

It follows then that $e_{x}\left(x, y_{j}\right)$ is a Legendre polynomial of degree 2. As such, it vanishes at the 2 Gauss points $x_{i}^{\ell 1}, x_{i}^{\ell 2}$ of $\left(x_{i}, x_{i+1}\right)$. Hence,

$\widetilde{w}_{x}\left(x_{i}^{\ell 1}, y_{j}\right)=\widetilde{w}_{x}^{I}\left(x_{i}^{\ell 1}, y_{j}\right), \quad \widetilde{w}_{x}\left(x_{i}^{\ell 2}, y_{j}\right)=\widetilde{w}_{x}^{I}\left(x_{i}^{\ell 2}, y_{j}\right)$.

We further remark that on the horizontal line $y=y_{j}$ we have that $\theta_{1}^{I}$ and $\widetilde{w}_{x}$ are each a polynomial of degree 2 . Using (4.10) we see that they coincide at three points, and hence they coincide on the whole line. Hence we might rewrite (4.12) as

$\theta_{1}^{I}\left(x_{i}^{\ell 1}, y_{j}\right)=\widetilde{w}_{x}^{I}\left(x_{i}^{\ell 1}, y_{j}\right), \quad \theta_{1}^{I}\left(x_{i}^{\ell 2}, y_{j}\right)=\widetilde{w}_{x}^{I}\left(x_{i}^{\ell 2}, y_{j}\right)$.

With the same argument we deduce that $\theta_{1}^{I}(x, y)-\widetilde{w}_{x}^{I}(x, y)$ also vanishes at the Gauss points of $y=y_{j+1}$ and $y=y_{j}^{m}$. For $\theta_{2}^{I}$ the same argument applies on the vertical edges, using $e_{y}\left(x_{i}, y\right)=\widetilde{w}_{y}\left(x_{i}, y\right)-\widetilde{w}_{y}^{I}\left(x_{i}, y\right)$, so that

$\Pi \boldsymbol{\theta}^{I}=\nabla \widetilde{w}^{I}$,

and the proof is concluded. 
Remark 4.2 The construction of $\boldsymbol{\theta}^{I}$ and $w^{I}$ (crucial for establishing the final estimates) could have been made also by more traditional techniques (i.e., through the "Stokes + Raviart-Thomas" approach). This however would have required the use of the $\mathbb{Q}_{2}-\mathbb{Q}_{1}$ Stokes element. The analysis of this element is not simple, and in general requires restrictions on the geometry of the elements (see [28]). Therefore we prefer the present construction because the extension to more general situations seems to have more possibilities.

In order to estimate the second piece of the right-hand side of (3.13) we make first two crucial observations.

The first one, quite obvious, is that $\Pi$ coincides with the identity operator when applied to constant vectors. For every $\eta \in \boldsymbol{\Theta}^{h}$ we can then denote by $\bar{\eta}$ its piecewise $L^{2}$ projection on constant vectors, and adding and subtracting $\bar{\eta} \equiv \Pi \overline{\boldsymbol{\eta}}$, and using (3.9) and usual approximation results we have

$$
\begin{aligned}
\|\boldsymbol{\eta}-\Pi \boldsymbol{\eta}\|_{0}= & \|(\boldsymbol{\eta}-\overline{\boldsymbol{\eta}})+\Pi(\overline{\boldsymbol{\eta}}-\boldsymbol{\eta})\|_{0} \leq\|(\boldsymbol{\eta}-\overline{\boldsymbol{\eta}})\|_{0} \\
& +\|\Pi(\overline{\boldsymbol{\eta}}-\boldsymbol{\eta})\|_{0} \lesssim h\|\boldsymbol{\eta}\|_{1} .
\end{aligned}
$$

The second observation is that for every $\eta \in \boldsymbol{\Theta}^{h}$ and for every $K \in \mathcal{T}_{h}$ the first component $\eta_{1}$ is a polynomial in $\mathbb{Q}_{2}$ while $(\Pi \eta)_{1}$ is a polynomial of $\mathbb{Q}_{1,2}$ that coincides with $\eta_{1}$ at the two Legendre points of the horizontal lines $y=y_{j}$, $y=y_{j+1}$ and $y=y_{j}^{m}$. Hence, on each of the vertical lines $x=x_{i}^{\ell 1}$ and $x=x_{i}^{\ell 2}$ the two polynomials $\eta_{1}$ and $(\Pi \eta)_{1}$ (as polynomials of degree two that coincide at three different points) coincide on the whole line. Consequently, by applying two-point Gauss integration in $x$, and Simpson's rule in $y$, we immediately see that

$$
\begin{aligned}
\int_{K} \eta_{1}(x, y)-(\Pi \eta)_{1}(x, y) \mathrm{d} x \mathrm{~d} y= & \int_{y_{j}}^{y_{j+1}} \mathrm{~d} y \int_{x_{i}}^{x_{i+1}} \eta_{1}(x, y) \\
& -(\Pi \eta)_{1}(x, y) \mathrm{d} x=0 .
\end{aligned}
$$

The same arguments obviously apply to the second component, so that we can write

$$
\int_{K} \eta(x, y)-(\Pi \eta)(x, y) \mathrm{d} x \mathrm{~d} y=0 .
$$

We can now easily estimate the second term of (3.13).

Theorem 4.3 Let $\left(U_{0}, \lambda_{0}\right) \equiv\left(\left(\boldsymbol{\theta}_{0}, w_{0}\right), \lambda_{0}\right)$ be the solution of (2.33). Then for every $V^{h}=(v, \eta) \in \mathcal{U}^{h}$ we have

$$
\left|\left(\lambda_{0}, B\left(V^{h}\right)-\Pi\left(B\left(V^{h}\right)\right)\right)_{0}\right| \lesssim h^{2}\left\|\lambda_{0}\right\|_{1, \Omega}\left\|V^{h}\right\|_{\mathcal{U}} .
$$

Proof We begin by recalling that from (2.24) we have $B\left(V^{h}\right)=\nabla v-\eta$, and from (3.8) we have $\Pi(\nabla v)=\nabla v$, so that

$$
B\left(V^{h}\right)-\Pi\left(B\left(V^{h}\right)\right)=-\eta+\Pi(\eta) .
$$

Hence, introducing $\overline{\lambda_{0}}$ as the piecewise constant mean value of $\lambda_{0}$, using (4.16) and Cauchy-Schwarz, and then usual approximation results and (4.15) we have

$$
\begin{aligned}
& \left|\int_{\Omega} \lambda_{0}(\boldsymbol{\eta}-\Pi \eta) \mathrm{d} x \mathrm{~d} y\right| \\
& \quad=\left|\int_{\Omega}\left(\lambda_{0}-\overline{\lambda_{0}}\right)(\boldsymbol{\eta}-\Pi \boldsymbol{\eta}) \mathrm{d} x \mathrm{~d} y\right| \\
& \leq\left\|\boldsymbol{\lambda}_{0}-\overline{\lambda_{0}}\right\|_{0, \Omega}\|\boldsymbol{\eta}-\Pi \boldsymbol{\eta}\|_{0, \Omega} \lesssim h^{2}\left\|\boldsymbol{\lambda}_{0}\right\|_{1, \Omega}\|\boldsymbol{\eta}\|_{1, \Omega} .
\end{aligned}
$$

Collecting the result of Theorem 3.3 together with the results of Theorems 4.1 and 4.3 we now have the final result.

Theorem 4.4 Let $\left(U_{0}, \lambda_{0}\right) \equiv\left(\left(w_{0}, \boldsymbol{\theta}_{0}\right), \lambda_{0}\right)$ be the solution of (2.33), and let $U_{0}^{h}$ be the solution of (3.12). Then we have

$\left\|U_{0}-U_{0}^{h}\right\|_{\mathcal{U}} \lesssim h^{2}\left(\left\|w_{0}\right\|_{3, \Omega}+\left\|\lambda_{0}\right\|_{1, \Omega}\right)$.

Therefore the element should behave optimally in the numerical plate solution considered in Fig. 1 and this is indeed the case as shown in Fig. 2.

\section{Concluding remarks}

Our objective in this paper was to give a mathematical analysis of the MITC9 shell element when used in plate bending solutions. Shell elements are used in general to model plate structures in engineering and the sciences; hence the analysis pursued herein is of considerable interest. While we considered a simplified setting, namely a clamped plate problem solved using uniform meshes and the limit problem with plate thickness equal to zero, the analysis is valuable because it gives insight into the behavior of the element.

The mathematical convergence analysis given in the paper shows that in this simple setting the element behaves optimally for displacements and rotations and hence does not lock. Some numerical results given in the paper also illustrate that it is reasonable to consider the case of vanishing plate thickness for the mathematical analysis.

Considering future work, since we used in all our analyses uniform meshes, it would be of value to also study the performance of the element in non-uniform decompositions, as well as the case of positive thickness. Furthermore, a more general mathematical convergence analysis of the MITC9 shell element when used for the solution of actual shell problems, that is, involving curved thin structures, would be very valuable. 


\section{References}

1. Bathe KJ (1996) Finite element procedures. Prentice Hall, New York

2. Zienkiewicz OC, Taylor RL (2005) The finite element method. Butterworth-Heinemann, Oxford

3. Brezzi F, Bathe KJ, Fortin M (1989) Mixed-interpolated elements for Reissner/Mindlin plates. Int J Num Methods Eng 28:1787-1801

4. Brezzi F, Fortin M (1991) Mixed and hybrid finite element methods. Springer, New York

5. Bathe KJ, Brezzi F, Cho SW (1989) The MITC7 and MITC9 plate bending elements. Comput Struct 32:797-814

6. Bathe KJ, Bucalem M, Brezzi F (1990) Displacement and stress convergence of the MITC plate bending elements. J Eng Comput 7(4):291-302

7. Chapelle D, Bathe KJ (1998) Fundamental considerations for the finite element analysis of shell structures. Comput Struct 66:19-36, 711-712

8. Chapelle D, Bathe KJ (2011) The finite element analysis of shellsfundamentals, 2nd edn. Springer, New York

9. Lee PS, Bathe KJ (2002) On the asymptotic behavior of shell structures and the evaluation in finite element solutions. Comput Struct 80:235-255

10. Lee PS, Bathe KJ (2005) Insight into finite element shell discretizations by use of the basic shell mathematical model. Comput Struct 83:69-90

11. Blouza A, Brezzi F, Lovadina C (1991) On the classification of linearly elastic shells. CRAS Série I 328:831-836

12. Baiocchi C, Lovadina C (2002) A shell classification by interpolation. Mat Mod Methods Appl Sci 12:1359-1380

13. Beirão da Veiga L (2003) Asymptotic energy behavior of two classical intermediate benchmark shell problems. Mat Mod Methods Appl Sci 13:1279-1302

14. Beirão da Veiga L, Chinosi C (2004) Numerical evaluation of the asymptotic energy behavior of intermediate shells with application to two classical benchmark tests. Comput Struct 82:525-534

15. Beirão da Veiga L (2005) Asymptotic study of the solution for pinched cylindrical shells. Comput Methods Appl Mech Eng 194:1113-1139
16. Hiller JF, Bathe KJ (2003) Measuring convergence of mixed finite element discretizations: an application to shell structures. Comput Struct 81:639-654

17. Bathe KJ, Lee PS (2010) Measuring the convergence behavior of shell analysis schemes. Comput Struct (in press)

18. Kardestuncer H (ed) (1987) Finite element handbook. McGraw-Hill, New York

19. Bucalem M, Bathe KJ (2011) The mechanics of solids and structures-hierarchical modeling and the finite element solution. Springer, New York (in press)

20. Bathe KJ (2009) The finite element method. In: Wah B (ed) Encyclopedia of computer science and engineering. Wiley, New York, pp 1253-1264

21. Lee PS, Bathe KJ (2010) The quadratic MITC plate and MITC shell elements in plate bending. Adv Eng Softw 41:712-728

22. Bathe KJ, Lee PS, Hiller JF (2003) Towards improving the MITC9 shell element. Comput Struct 81:477-489

23. Bucalem M, Bathe KJ (1993) Higher-order MITC general shell elements. Int J Numer Methods Eng 36:3729-3754

24. Iosilevich A, Bathe KJ, Brezzi F (1997) On evaluating the inf-sup condition for plate bending elements. Int $\mathrm{J}$ Numer Methods Eng 40:3639-3663

25. Chapelle D, Bathe KJ (2010) On the ellipticity condition for model-parameter dependent mixed formulations. Comput Struct 88:581-587

26. Brezzi F, Fortin M, Stenberg R (1991) Error analysis of mixedinterpolated elements for Reissner-Mindlin plates. Math Model Methods Appl Sci 1:125-151

27. Brezzi F, Evans JA, Hughes TJR, Marini LD (2010) New quadrilateral plate elements based on Twist-Kirchhoff theory. Comput Methods Appl Mech Eng (submitted)

28. Stenberg R (1981) Blandade finita elementmetoder för två problem inom strömningsmekaniken och hållfathetsläran. Licentiatarbete, Department of Technical Physics and Mathematics, Helsinki University of Technology 